\title{
Unerupted Canine and Premolars Width Estimation Based on Bayesian Approach: A Comparative Study
}

\author{
Allahyar Geramy, ${ }^{1}$ and Maryam Moghaddas Bayat ${ }^{2,}$ \\ ${ }^{1}$ Department of orthodontics, Tehran University of Medical Sciences, Tehran, IR Iran \\ ${ }^{2}$ Econometrician, Tehran, IR Iran \\ "Corresponding author: Maryam Moghaddas Bayat, Tehran, IR Iran. Tel: +98-9120286467, E-mail: moghaddasbayat.maryam@gmail.com
}

Received 2016 May 09; Accepted 2016 June 10.

\begin{abstract}
Background: During mixed dentition period, one can make accurate estimation of future dental development and can assess whether there will be enough space in the dental arch. In orthodontics treatment planning, it is vital to predict space required for unerupted canine and premolars in the arch.

Objectives: The main goal of this study is to compare different teeth combinations in predicting needed space for unerupted canine and premolars on Bayesian approach and introduce the most reliable one.

Patients and Methods: The sample for this study consists of 47 dental casts (19 males, 28 females) with complete erupted dental arches. The meisodistal width of all teeth was measured using a dental caliper. We consider different combinations of teeth size and compare them to find the best predictor. In order to do that, quantile regression and Bayesian approach are applied using " $\mathrm{R}$ software".

Results: Combination of first maxillary molars with sum of central and lateral mandibular incisors has the smallest standard deviation. This is true for male and female samples. The regression formula based on this teeth combination has been introduced.

Conclusions: In our sample, combination of Mandibular incisors and maxillary first molar is found to be better than the other predictors for female and female model in both arches.
\end{abstract}

Keywords: Mixed Dentition Analysis, Unerupted Canine And Premolars, Quantile Regression, Bayesian Approach

\section{Background}

Dental occlusion is the way that maxillary and mandibular teeth contact each other. Main therapeutic aim for orthodontic treatment is provide patients with ideal/ normal dental occlusion. Malocclusion is any deviation from regular occlusion. Mostly, the deviation is a result of an imbalance between teeth size and arch size. Most of the problems arise during mixed dentition period. The problem can be reduced or even removed entirely if it is managed appropriately.

During mixed dentition period, one can make accurate estimation of future dental development and can assess whether there will be enough space in the dental arch to let all teeth erupt. In orthodontics treatment planning, it is vital to predict space required for unerupted canine and premolars in the arch. The prediction of tooth size must be done before eruption by mixed dentition analysis. In mixed dentition space analysis, mesiodistal width of unerupted canine and premolars can be predicted by different methods, one of which is using a regression model.

Since correct orthodontic diagnosis and treatment planning is based on the prediction, accurate prediction is vital. In this regards, modeling equation by using size of already erupted permanent teeth data is the most widely used method. Tooth size varies in different national groups and among genders. Consequently, prediction equation must be specified to each population individually.

To the best of our knowledge, linear regressions are used to specify the equation. Standard linear regression summarizes the average relationship between a set of predictors and the response variable. Predictor coefficient represents the change in the mean value of dependent (response) varaible given one unit change in predictor. A single slope is used to describe the relationship. Therefore, linear regression provides a partial view of the link between the response variable and predictors. This is often inadequate when there is heterogeneous variance between predictor and dependent variable. In such cases, we need to examine how the relationship between predictor and dependent variable changes depending on the value of dependent variable. For example, the effect of parental care on mean infant birth weight can be compared to its ef- 
fect on other quantiles of infant birth weight. Quantile regression solves for these problems by looking at changes in the different quantiles of the response. The parameter estimates for this technique represent the change in a specified quantile of the response variable produced by one unit change in the predictor variable. One major benefit of quantile regression is that it makes no assumptions about the error distribution. This article uses quantile regression and Bayesian approach to conduct the mixed dentition space analysis.

Hixon and Oldfather (1) model a regression equation to predict size of unerupted mandibular canine and premolars by using radiographic measurement of mandibular premolars.

The method is modified by Stahle (2) via including size of the mandibular permanent incisor. Staley and Kerber (3) revise Hixon and Oldfather method (1). They develop the equation and make a graph for the estimation of mesiodistal width of unerupted canine and premolars. Moyers (4) establishes two probability tables to predict mesiodistal width of unerupted canine and premolars during mixed dentition based on the sum of mesiodistal widths of four mandibular permanent incisors. The tables are the most widely used for mixed dentition because of the following advantages: i) minimal systematic error. ii) less time consuming iii) applicable to both upper and lower dental arches.

Tanaka and Johnston (5) model a probability equation to calculate mesiodistal width of unerupted canine \& premolars by sum of four mandibular incisors data. This method is simple and applicable for both dental arches and genders. The model has also been used widely with a standard accuracy. It is worth to notice that Moyers and also Tanaka and Johnston method has been studied for population of other racial and ethnic origins (6-10).

Many authors reported that sex factor should also be included in probability equation as an extra predictor for the estimation of unerupted canine and premolars. So regression equation or tables must be made detached for both males and females $(11,12)$.

On one hand, sum of mesiodistal width of four mandibular incisors has been most widely used to advance regression equation in order to predict size of unerupted canine \& premolars by many researchers. (13-17). On the other hand, applicability of Moyers and Tanaka method has been tested by some researchers and they found that these methods do not truly predict unerupted canine and premolars space in their sample (17).

Therefore, some researchers have used different combinations of other erupted permanent teeth in their study. In this regards, Cattaneo et al. (18) study combination of teeth including four mandibular incisors, mandibular incisors and mandibular first molar and also mandibular incisors and maxillary first molar. They conclude that sum of four mandibular incisors and maxillary first molar is the best predictor for estimating mesiodistal width of unerupted permanent canine and premolars in their sample.

Nourallah (19) validate Tanaka and Johnston's analysis on Syrian patients. Tanaka and Johnston's tables and equations are modified in order to improve the precision of the prediction. The correlation coefficients found between the permanent mandibular central incisors space and maxillary first molars and the maxillary and mandibular canines and premolars are high. New prediction tables applicable at earlier ages, and new regression equations are constructed. Galvao et al. (20) based on the literature available and their systematic review, conclude that the Moyers mixed dentition analysis must be carefully used, since the majority of the articles analyzed showed that the probability of $75 \%$ was not as accurate as expected, leading to the need of adapting the probability levels depending on the study population.

\section{Objectives}

This article has two following goals:

I. Using Bayesian approach capabilities to develop estimation procedures.

II. Using different combinations of teeth size and comparing them in order to find the best predictor.

\section{Patients and Methods}

The sample for this study consists of 47 dental casts (19 males, 28 females) with complete eruption of permanent mandibular incisors, canines, molars and premolars, as well as maxillary ones. The sample is selected from population of patients seen at department of orthodontics, Tehran University of medical sciences. All individuals selected for this study are between 11 and 25 years without prior orthodontic treatment.

To meet the goals and regarding our sample size, we select quantile regression by means of Bayesian approach based on following reasons.

When estimating regression models, we have to be aware of the implications of model assumptions when interpreting the results. Symmetry, linearity, and variance homogeneity are among the strongest but common assumptions. The classical theory of linear models is basically a theory for models of conditional expectations. In many applications, it is fruitful to go beyond these models. Quantile regression is gradually developing as a comprehensive approach to the statistical analysis of linear and 
nonlinear response models. A number of papers have recently performed on the application of quantile regression. Quantile regression is an intellectually stimulating and practically very useful framework where such assumptions can be relaxed.

More clearly, Quantile regression is a statistical technique intended to estimate, and conduct inference about conditional quantile functions. This technique provides a more complete statistical analysis of the stochastic relationships among random variables. In other words, quantile regression allows analyst to study the impact of independent variables $(\mathrm{X})$ on different quantiles of dependent variable's $(\mathrm{Y})$ distribution, and thus gives us a complete picture of the relationship between $Y$ and X. Therefore, estimation and inference are distribution-free. Moreover, quantile regression is robust to outliers in y observations.

The use of Bayesian inference in generalized linear and additive models is quite standard nowadays. The relative comfort with which Markov chain Monte Carlo (MCMC) methods may be used for obtaining the posterior distributions, even in complex circumstances, has made Bayesian inference very beneficial and attractive. Unlike conventional methods, Bayesian inference delivers one with the whole posterior distribution of the parameter of interest. In addition, it takes to be account parameter uncertainty when making predictions. However, in the area of quantile regression, there is very little in the literature along the Bayesian inference lines.

Recent work by Schennach (21) has opened the way to a Bayesian treatment of quantile regression. This method provides a likelihood for data "y" subject only to a set of $\mathrm{m}$ moment conditions of the form $\operatorname{Eg}(\mathrm{y}, \theta)=0$, where $\theta$ is a k dimensional parameter of interest. The method make a likelihood supported on the $n$ data points that is minimally informative, in the sense of maximum entropy, subject to the moment conditions. Specifically the probabilities $\{\mathrm{pi}\}$ attached to the $\mathrm{n}$ data points are chosen to solve following problem.

$\max _{p} \Sigma_{i=1}^{n}-$ pilogpi

The solutions of this problem, well known in the maximum entropy literature, e.g. Jaynes (22).

$\operatorname{pi}(\theta)=\frac{\exp \left\{\lambda(\theta)^{\prime} g(\mathrm{yi}, \theta)\right\}}{\sum_{i=1}^{n} \exp \left\{\lambda(\theta)^{\prime} g(\mathrm{yi}, \theta)\right\}}$

The resulting likelihood for i.i.d data is:

$\prod_{i=1}^{n} \mathrm{pi}(\theta)$

This may be combined with a prior density on $\theta$ to yield the posterior density:

$p(\theta \mid Y)=p(\theta) \prod_{i=1}^{n} \operatorname{pi}(\theta)$
Posterior odds ratio is used to choose the best prediction equation. The formula is as follows.

$$
\begin{aligned}
\mathrm{POR} & =\frac{\operatorname{pr}\left(H_{1} \mid D\right)}{\operatorname{pr}\left(H_{0} \mid D\right)} \\
& =\frac{\operatorname{pr}\left(D \mid H_{1}\right) / \operatorname{pr}\left(\mathrm{H}_{1}\right) /}{\operatorname{pr}\left(D \mid H_{0}\right) / \operatorname{pr}\left(\mathrm{H}_{0}\right)}
\end{aligned}
$$

Where, Equation 6 is defined.

$$
\begin{aligned}
& p\left(H_{0} \mid D\right)=1-p\left(H_{1} \mid D\right) \\
& \quad \operatorname{ppr}\left(\mathrm{H}_{0}\right)
\end{aligned}
$$

Moreover, the asymmetric Laplace distribution is used for Bayesian quantile regression.

\section{Results}

The statistical characteristics includes range, standard deviation and skewness of variables distributions are presented in Table 1 . Table 1 shows tooth group measurements which are determined by summing the mesiodistal dimensions of the teeth within the group. The predictors consist of left and right mandibular teeth as well as maxillary ones. The predictor variables entail sum of central and lateral mandibular incisors first maxillary molars in addition to sum of central and lateral mandibular incisors, and central incisors in both arch. The dependent variable comprises sum of canine and premolars in both arch.

Table 1 shows that combination of first maxillary molars with sum of central and lateral mandibular incisors has smallest standard deviation. This is true among male and female sample. In addition, dependent variable distribution of maxillary arch is positively skewed. While, dependent variable distribution of mandibular arch is negatively skewed. This is true within male and female data.

This research valuated the effect of sex in the dentition analysis. To do that, we implement a consistent integrated squared difference test for investigating equality of densities between male and female data .The number of bootstrap replications in test is 99 . Table 2 confirms that equality can be rejected at 5 percent level. So, two different regressions for male and female data are needed in dentition analysis.

In addition, this study uses different combinations of teeth size as predictor for the estimation. Then, the alternative predictors are compared to find the best predictor. The comparisons implement via Bayes factor. Results are presented in Table 3.

According to Table 2 and Table 3, findings can be summarized as follows:

1. Significant differences are found for males and females estimation. 


\begin{tabular}{|c|c|c|c|c|c|c|c|c|}
\hline \multirow{3}{*}{$\begin{array}{l}\text { Tooth } \\
\overline{21 \mid 12}\end{array}$} & \multicolumn{4}{|c|}{ Female } & \multicolumn{4}{|c|}{ Male } \\
\hline & \multicolumn{2}{|c|}{ Range } & \multirow{2}{*}{$\begin{array}{r}\mathrm{SD} \\
1.342\end{array}$} & \multirow{2}{*}{$\begin{array}{l}\text { Skewness } \\
\text { - } 0.401\end{array}$} & \multicolumn{2}{|c|}{ Range } & \multirow{2}{*}{$\begin{array}{c}\text { SD } \\
1.106\end{array}$} & \multirow{2}{*}{$\begin{array}{r}\text { Skewness } \\
-0.305\end{array}$} \\
\hline & 19.44 & -25.40 & & & 19.50 & -24.32 & & \\
\hline $345 \mid 345$ & 18.23 & -24.33 & 1.213 & -0.050 & 19.50 & - 24.32 & 1.022 & -0.085 \\
\hline $345 \mid 345$ & 19.14 & -24.42 & 1.185 & 0.131 & 19.75 & -24.62 & 1.148 & 0.129 \\
\hline$\frac{1 \mid 1}{1 \mid 1}$ & 24.87 & - 30.92 & 1.654 & -0.227 & 25.62 & -30.52 & 1.366 & -0.540 \\
\hline$\underline{6} \overline{21 \mid 12} \underline{6}$ & 19.28 & -24.03 & 1.117 & -0.080 & 20.01 & -24.17 & 0.982 & 0.048 \\
\hline
\end{tabular}

Table 1. Range, Standard Deviation, and Skewness of The Variables

2. The best predictor in female equation of maxillary and mandibular arches is combination of first maxillary molar in addition to sum of central and lateral mandibular incisors.

3. The best predictor in male equation of maxillary arch is combination of first maxillary molar in addition to sum of central and lateral mandibular incisors. While in male equation of mandibular arch, there is no superiority of combination first maxillary molar in addition to sum of central and lateral mandibular incisors and combination of mandibular incisors.

Accordingly, we specify the male and female model including combination of first maxillary molar in addition to sum of central and lateral mandibular incisors for both arches. Considering standard regression is specified under some extremely simplifying assumptions like linearity and variance homogeneity, we drop such assumptions in quantile regression specification.

Figures 1 - 4 show quantile regression plot. The plot shows a scatterplot of sum of canine premolars in both arches on best predictors for male and female model. Superimposed on the plot are the ).05,.25,.5,.75,.95 (quantile regression lines as the dashed line, and the least squares estimate of the conditional mean function as solid line which is close to median fit.

Figures 1 - 4 shed new light on the data, which implies that:

1. Estimating mesiodistal width of unerupted canine
Table 2. Consistent Density Equality Test

\begin{tabular}{lc}
\hline & PValue \\
\hline Maxillary densities & 0.0202 \\
Mandibular densities & 0.0303 \\
\hline
\end{tabular}

and premolars obtained from an estimate under normality assumption on one hand and quantile regression on the other hand are hardly dramatic for the data.

2. Considering obtained results, we can conclude that the whole distribution of mesiodistal width of unerupted canine and premolars changes with predictors and that assumptions like symmetry and variance homogeneity might be questionable for such type of analysis according to our sample.

Therefore, we find quantile regression choice appropriate for Mixed Dentition Space Analysis in our sample. Due to the results and according to the sample, we choose Bayesian regressions of 0.5 and 0.25 quantiles for male and female models which are presnted in Table 4.

\section{Discussion}

Difference between available and required spaces in the arch can be predicted during mixed dentition period. This helps to determine whether enough space is available for posterior teeth in the arch, so that they can erupt 


\begin{tabular}{|c|c|c|}
\hline & & \\
\hline \multirow{2}{*}{ Male } & $\left(\frac{1 \mid 1}{1 \mid 1}\right)$ vs. $(\overline{21 \mid 12})$ & $(\overline{21 \mid 12})$ vs. $(\underline{6} \overline{12 \mid 12} \underline{6})$ \\
\hline & 0.2617 & 0.6098 \\
\hline \multirow{3}{*}{ Female } & $\left(\frac{1 \mid 1}{1 \mid 1}\right)$ vs. $(\overline{21 \mid 12})$ & $(\overline{21 \mid 12})$ vs. $(\underline{6} \overline{12 \mid 12} \underline{6})$ \\
\hline & 0.0242 & 0.0072 \\
\hline & \multicolumn{2}{|c|}{ Mandibular Arch } \\
\hline \multirow{2}{*}{ Male } & $\left(\frac{1 \mid 1}{1 \mid 1}\right)$ Vs. $(\overline{21 \mid 12})$ & $(\underline{6} \overline{12 \mid 12} \underline{6})$ vs. $(\overline{21 \mid 12})$ \\
\hline & $1.148 \times 10^{-9}$ & 1 \\
\hline \multirow{2}{*}{ Female } & $\left(\frac{1 \mid 1}{1 \mid 1}\right)$ vs. $(\overline{21 \mid 12})$ & $(\overline{21 \mid 12})$ vs. $(\underline{6} \overline{12 \mid 12} \underline{6})$ \\
\hline & 0.0002 & 1.424 \\
\hline
\end{tabular}

Table 3. Comparison Test Between Alternative Predictors Via Bayes Factor

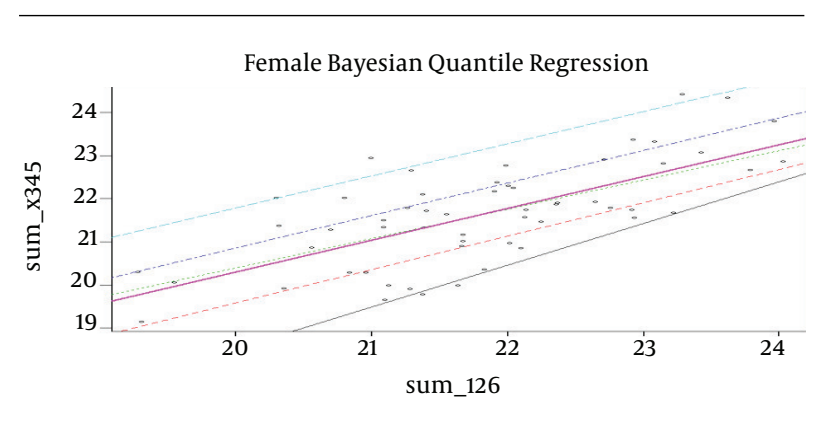

Figure 1. Mesiodistal Width of Unerupted Maxillary Canine and Premolars Estimation for Female by Using Sum of 1st Maxillary Molar in Addition to Sum of Central and Lateral Mandibular Incisors as Predictor

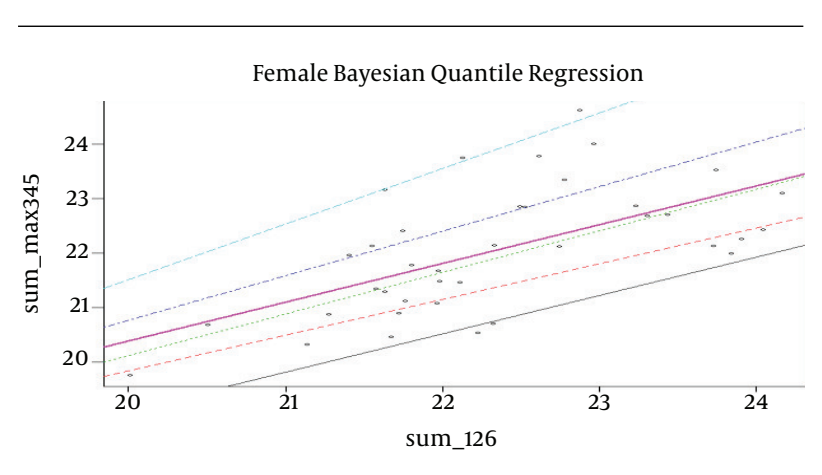

$\overline{\text { Figure 2. Mesiodistal Width of Unerupted Maxillary Canine and Premolars Estima- }}$ tion for Male by Using Sum of 1st Maxillary Molar in Addition to Sum of Central And Lateral Mandibular Incisors as Predictor.

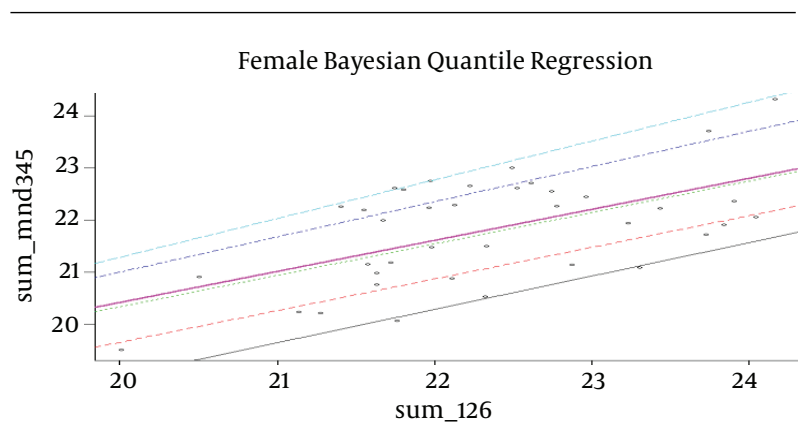

Figure 3. Mesiodistal Width of Unerupted Mandibular Canine and Premolars Estimation for Female by Using Sum of 1st Maxillary Molar in Addition to Sum of Central and Lateral Mandibular Incisors as Predictor

freely with good alignment. The analysis is very important regarding orthodontic diagnosis and treatment planning. Prediction equations based on mesiodistal width of already erupted permanent teeth is used to predict size of unerupted canine and premolars.

This research tries to specify a model which is relatively noninvasive and accurate applicable for both dental arches and genders. In order to do that, this study uses combination of teeth sizes encompasses mandibular incisors alone, combination of mandibular incisors and maxillary first molar and sum of central incisors in both arch as predictor to estimate the mesiodistal width of unerupted canine and premolars in Iranian sample. It is worth mentioning that, the mentioned group of teeth were most frequently 


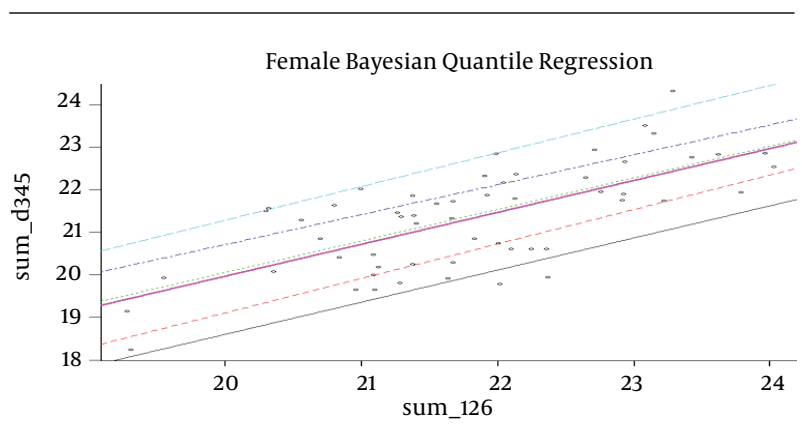

Figure 4. Mesiodistal Width of Unerupted Mandibular Canine and Premolars Estimation for Male by Using Sum of 1st Maxillary Molar in Addition to Sum of Central and Lateral Mandibular Incisors As Predictor

Table 4. Bayesian Quantile Regression ${ }^{\mathrm{a}}$

\begin{tabular}{lcc}
\hline & Intercept & Coefficient \\
\hline & Mandibular Arch Equation \\
\hline Female & 5.434 & 0.732 \\
\hline Male & 7.284 & 0.612 \\
\hline & Maxillary Arch Equation & \\
\hline & 6.832 & 0.679 \\
\hline Female & 4.969 & 0.758 \\
\hline Male & $\begin{array}{l}\text { a } \\
\text { the equation is defined: (predicted space for unerupted canine and premolar) } \\
\text { = intercept }+ \text { coefficient } \times \text { (combination of first maxillary molar in addition to } \\
\text { sum of central and lateral mandibular incisors). }\end{array}$
\end{tabular}

applied in different populations earlier.

Our sample includes male and female data. This research valuated the effect of sex in the mixed dentition analysis. Regression equations are generated by the method of Bayesian quantile regression. Applying Bayesian quantile regression to the raw data (for males, females, the combination predictors, maxillary and mandibular arches), 12 equations are obtained. Then, a statistical test is applied to determine significant difference between males and females for the tooth groups. Significant differences are found for males and females. Consequently, the estimation include different equation for males and females.

In addition, equations are compared based on Bayes factor to determine the best predictor for dependent variable. Results implies that sum of central incisors in both arch is not validated for estimation of mesiodistal width of canine and premolar among above mentioned predictor on the sample. However, combination of Mandibular incisors and maxillary first molar is found to be better than the others for male and female model in both arches.

Given the results from Bayesian quantile regression, different intercept and coefficient in the quantiles for both arches are obtained. If one looks more carefully at the results, one sees interesting departures from classical assumptions that standard regression is specified under those. This is only due to the assumption violation by the data. Hence, it would not be likely to be revealed the statistical features of dependent variable by such misspecifications. One common remedy for symptoms like these would be to reformulate the model in other method that relaxes the assumptions.

\subsection{Conclusion}

We found that Bayesian quantile regression is an appropriate method for determining tooth size in our sample. It should be stressed that other researches are suggested to conduct large scale study on a local population sample to evaluate accuracy of our results for prediction unerupted canine and premolars width.

\section{Footnote}

Authors' Contribution: Dental part by Allahyar Geramy and The analysis part by Maryam Moghaddas Bayat.

\section{References}

1. Hixon EH, Oldfather RE. Estimation of the sizes of unerupted cuspid and bicuspid teeth. Angle Orthod. 1958;28(4):236-40.

2. Stahle H. Determination of mesiodistal crown width of the unerupted permanent cuspids and bicuspids. Helv Orthod Acta. 1959;3:14-9.

3. Staley RN, Kerber PE. A revision of the Hixon and Oldfather mixeddentition prediction method. Am J Orthod. 1980;78(3):296-302. [PubMed: 6931493].

4. Moyers RE. Handbook of orthodontics. 4 ed. chicago: year book medical publisher; 1958.

5. Tanaka MM, Johnston LE. The prediction of the size of unerupted canines and premolars in a contemporary orthodontic population. JAm Dent Assoc. 1974;88(4):798-801. [PubMed: 4525402].

6. Al-Bitar ZB, Al-Omari IK, Sonbol HN, Al-Ahmad HT, Hamdan AM Mixed dentition analysis in a Jordanian population. Angle Orthod. 2008;78(4):670-5. [PubMed: 18302466].

7. Alessandri Bonetti G, Verganti S, Zanarini M, Bonetti S, Gatto MR. Mixed dentition space analysis for a northern Italian population: new regression equations for unerupted teeth. Prog Orthod. 2011;12(2):949. [PubMed: 22074832].

8. Bolton WA. Disharmony in tooth size and its relation to the analysis and treatment of malocclusion. Angle Orthod. 1958;28(3):113-30.

9. Brook AH, Griffin RC, Townsend G, Levisianos Y, Russell J, Smith RN. Variability and patterning in permanent tooth size of four human ethnic groups. Arch Oral Biol. 2009;54 Suppl 1:S79-85. [PubMed: 19144325].

10. Yuen KK, Tang EL, So LL. Mixed dentition analysis for Hong Kong Chinese. Angle Orthod. 1998;68(1):21-8. [PubMed: 9503131].

11. Bernabe E, Flores-Mir C. Are the lower incisors the best predictors for the unerupted canine and premolars sums? an analysis of a Peruvian sample. Angle Orthod. 2005;75(2):202-7. [PubMed:15825783]. 
12. Hashim HA, Al-Shalan TA. Prediction of the size of un-erupted permanent cuspids and bicuspids in a Saudi sample: a pilot study.J Contemp Dent Pract. 2003;4(4):40-53. [PubMed:14625594].

13. Diagne F, Diop-Ba K, Ngom PI, Mbow K. Mixed dentition analysis in a Senegalese population: elaboration of prediction tables. Am J Orthod Dentofacial Orthop. 2003;124(2):178-83. [PubMed: 12923514].

14. Jaiswal AK, Paudel KR, Shrestha SL, Jaiswal S. Prediction of space available for unerupted permanent canine and premolars in a Nepalese population. J Orthod. 2009;36(4):253-9. [PubMed: 19934243].

15. Malkoc S, Basciftci FA, Nur M, Catalbas B. Maxillary and mandibular mesiodistal tooth sizes among different malocclusions in a sample of the Turkish population. Eur J Orthod. 2011;33(5):592-6. [PubMed: 21097991].

16. van der Merwe SW, Rossouw P, van Wyk Kotze TJ, Trutero H. An adaptation of the Moyers mixed dentition space analysis for a Western Cape Caucasian population. J Dent Assoc S Afr. 1991;46(9):475-9. [PubMed: 1820683].

17. Memon S, Fida M. Comparison of three mixed dentition analysis methods in orthodontic patients at AKUH. J Coll Physicians Surg Pak. 2010;20(8):533-7. [PubMed: 20688019].

18. Cattaneo C, Butti AC, Bernini S, Biagi R, Salvato A. Comparative evaluation of the group of teeth with the best prediction value in the mixed dentition analysis. EurJPaediatr Dent. 2010;11(1):23-6. [PubMed: 20359277].

19. Nourallah AW, Gesch D, Khordaji MN, Splieth C. New regression equations for predicting the size of unerupted canines and premolars in a contemporary population. Angle Orthod. 2002;72(3):216-21. [PubMed: 12071605].

20. Galvao M, Dominguez GC, Tormin ST, Akamine A, Tortamano A, de Fantini SM. Applicability of Moyers analysis in mixed dentition: A systematic review. Dental Press J Orthod. 2013;18(6):100-5. [PubMed: 24351156]

21. Schennach SM. Bayesian exponentially tilted empirical likelihood. Biometrika. 2005;92(1):31-46.

22. Jaynes ET. Probability theory: the logic of science. Cambridge, United Kingdom: Cambridge university press; 2003. 